\title{
The TUS orbital detector simulation
}

\author{
L.Tkachev* \\ Joint Institute for Nuclear Research, Dubna, Russia \\ E-mail: tkatchev@jinr.ru \\ A.Grinyuk, M. Lavrova \\ Joint Institute for Nuclear Research, Dubna, Russia
}

A.Tkachenko

Joint Institute for Nuclear Research, Dubna, Russia

Bogolyubov Institute for Theoretical Physics, Kiev, Ukraine

\begin{abstract}
The TUS space experiment is aimed to study energy spectrum and arrival distribution of UHECR at energy range above $10^{20} \mathrm{eV}$ by the measurement of the EAS fluorescent radiation in atmosphere. The TUS mission is scheduled for launching in late 2015 at the dedicated "Lomonosov" satellite. TUSSIM program package was developed to simulate the TUS detector performance including the Fresnel mirror optical parameters, the light concentrator of the photo detector, the front end and trigger electronics. In order to study the detector response, we employ the software package ESAF of JEM-EUSO experiment for the fluorescent radiation of EAS. Trigger efficiency is crucially dependent on the background level that is changed from $0.2 \cdot 10^{6}$ to $15 \cdot 10^{6} \mathrm{ph} /\left(\mathrm{m}^{2} \cdot \mu \mathrm{s} \cdot \mathrm{sr}\right)$ at moonless and full moon nights respectively. The TUSSIM algorithms is described and the expected TUS statistics is presented for 5 years of data collection from $500 \mathrm{~km}$ solar-sinchronized orbit taking into account the background light intensity change during the space flight.
\end{abstract}

The 34th International Cosmic Ray Conference,

30 July- 6 August, 2015

The Hague, The Netherlands

\footnotetext{
*Speaker.
} 


\section{Introduction}

The TUS project's task is the experimental study of Ultra High Energy Cosmic Rays (UHECR). The fluorescent and Cherenkov radiation of Extensive Air Showers (EAS) generated by UHECR particles is supposed to detect in the Earth atmosphere at the night side of the space orbit at altitudes $400-500 \mathrm{~km}$. It will be possible to measure the $\mathrm{CR}$ spectrum, composition and arrival directions at $E>7 \cdot 10^{19} \mathrm{eV}$ [1] beyond the GZK energy limit. The SINP MSU, JINR and Space Regatta Consortium together with several Korean and Mexican Universities collaborate in the TUS detector preparation.

Important advanage of this detector is a possibility to take data from the different arrival directions of the sky with the same apparatus and with the same systematics. Besides the atmospheric conditions is more stable for the EAS measurements from space orbit in comparison with ground based study. With such a data the existent difference between the results of Auger detector in the south hemisphere and TA detector in north one may be understood [2] including obtaining definite conclusions concerning anisotropy and mass composition of UHECRs. However the EAS signal at the space orbit is in 100 times weaker in comparison with the ground based detectors that leads to a difficult problem due to background albedo radiation of the Earth atmosphere. The permanent monitoring and calibration of the apparatus is supposed during all the TUS data taking in orbit [3] to get the reliable data and interpret them adequately.

The TUS detector is produced and the preflight tests are in progress. It will be the first detector in space for the UHECR study by the fluorescent EAS radiation measurement. The TUS mission is scheduled for launch at the end of 2015 aboard of the dedicated "Lomonosov" satellite, and is expected to operate for 3-5 years [4].

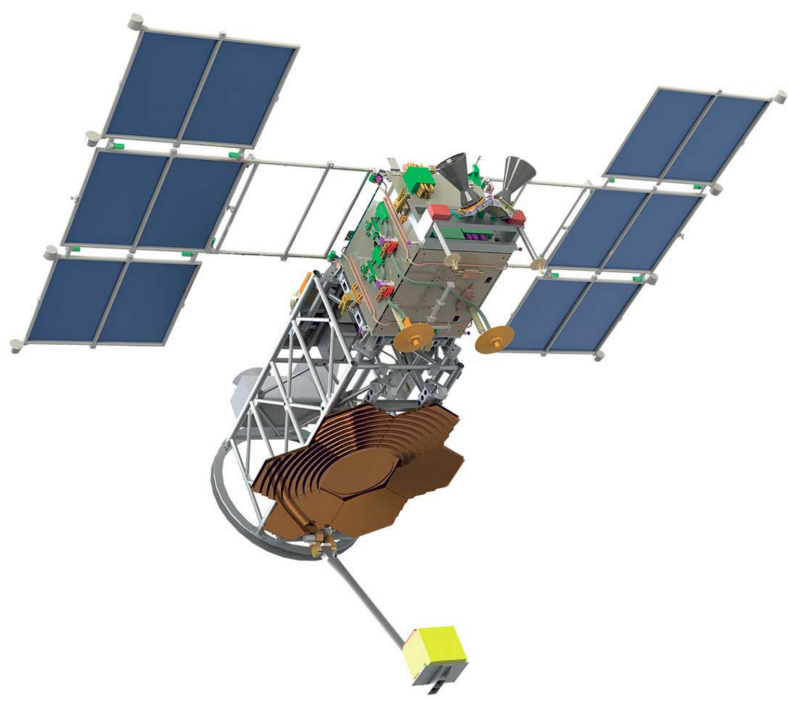

Figure 1: TUS Detector on-board the Lomonosov satellite. 


\section{The TUS detector and selection of UHECR events}

The EAS UV radiation is detected by the optical system of the TUS detector. There are two main parts of this detector as seen in Fig. 1: a modular Fresnel mirror and a photo receiver matrix with the corresponding DAQ electronics [5] (field-of-view (FoV) $\pm 4.5^{\circ}$, the number of pixels 16x16). One pixel means Hamamatsu type R1463 PMT with $13 \mathrm{~mm}$ tube diameter, multi-alcali cathode and UV glass window. The Fresnel mirror has area 1.8 $\mathrm{m}^{2}$ and the focal distance $1.5 \mathrm{~m}$. The DAQ electronics forms 256 channels with a time resolution (time step) of $\Delta t=0.8 \mu \mathrm{s}$. One pixel FOV is $\sim 0.1 \mathrm{msr}$ that corresponds to a spatial spot of $5 \times 5 \mathrm{~km}^{2}$ on the Earth surface for the $500 \mathrm{~km}$ orbit height and $80 \mathrm{x} 80 \mathrm{~km}^{2}$ for the whole detector.

The photodetector consists of the 16 clusters each of 16 pixels and the front end cluster boards. The cluster board regulates a HV tension on PMTs according to the background atmospheric light level and calculates so called Sliding sum (S-sum) $S S_{i j}(t)$ value - the sum of the PMT's amplitudes $A_{i j}$ during the last 16 time steps in each pixel

$$
S S_{i j}(t)=\sum_{0}^{15} A_{i j}(t-(15-k) \Delta t)
$$

where $\mathrm{i}, \mathrm{j}$ are the cluster and pixel numbers respectively. The front end trigger electronics elaborate the trigger signal of the level 1 in this cluster if the S-sum value is above of the prescribed threshold value $T$. Every 16 time steps the cluster boards transmits the S-sum values to the motherboard that forms a map of the $16 \times 16$ pixels where the S-sum value were above the threshold. Trigger of the level 2 is elaborated if in the $M$ consecutive maps the hitted pixels are localized in the same or neighbouring bins. In Fig. 2 an example of consecutive maps with the hitted pixels for one simulated event is presented. $T$ and $M$ values can be set from the mission center and depend on the atmospheric background level and the trigger rate that is limited by channel transver capacity to the on-board computer.
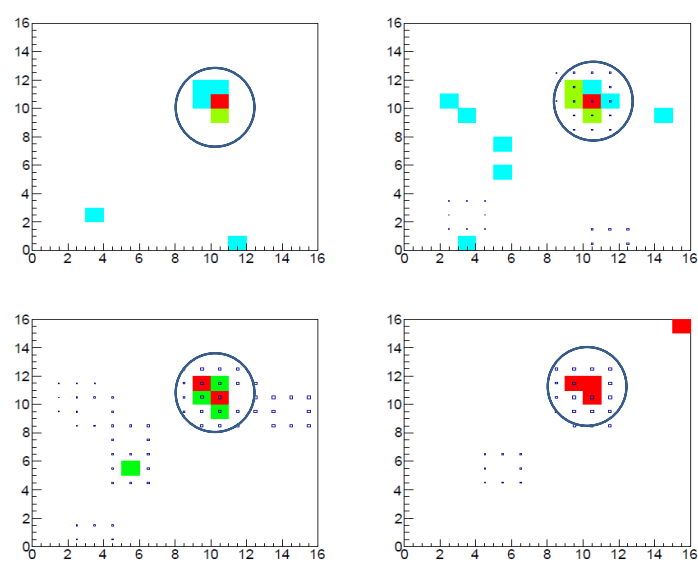

Figure 2: Consecutive maps with the hitted pixels for $E=\sim 10^{20} \mathrm{eV}$ event at $20 \%$ of background level and corresponding to the trigger rate of $\sim 100 \mathrm{ev} /$ minute. Pixels in circles form trigger if $M=3$ or $M=4$ trigger topology is established. 
At the trigger of the 2 level condition fulfilled the raw data array of 256 PMT currents for 256 time steps is transmitted to on-board computer from the motherboard memory. The EAS event size contains 80-100 Kbytes and trigger rate has to be 1-2 per minutes due to limited speed of the raw data transmition. Elaboration of the trigger 2 level demands $M=3 \div 5$ consecutive maps or $38.4 \div 64.0 \mu$ s. This means that it needs to generate $\sim 10^{6}$ simulated events to evaluate trigger efficiency at the 1-2 trigger rate per minute.

3. The TUS detector and electronics simulation

The TUSSIM program package was developed at JINR to simulate the TUS detector performance including the Fresnel mirror optical parameters, the light concentrator of the photo detector, the front end and trigger electronics to optimize the thigger efficency. In order to research the detector response, we employ the software package ESAF of JEMEUSO experiment [6] for the fluorescent radiation of EAS. The avarage EAS event duration is $\sim 100 \mu s$ with RMS $\sim 45 \mu s$ as it was obtained from ESAF simulation. Each photon falling on the TUS mirror was traced through the optical system taking into account the local optical parameters of the mirror model that was obtained according to the previous measurements [7].

As it will be shown the trigger efficiency is crucially dependent on the background level that changed according to the TATIANA satellite measurements [8] from $\sim 0.2 \cdot 10^{6}$ to $\sim 15 \cdot 10^{6} \mathrm{ph} /\left(\mathrm{m}^{2} \cdot \mu \mathrm{s} \cdot \mathrm{sr}\right)$ at moonless and full moon nights respectively with the avarage background light level $I_{\circ} \sim 2 \cdot 10^{6} \mathrm{ph} /\left(\mathrm{sr} \cdot \mu \mathrm{s} \cdot \mathrm{m}^{2}\right)$.

TUSSIM program simulates a procedure of the UHECR event selection by the trigger criteria of the level 1 and 2 as it described above. The background photons were generated homogeneously in the time steps according to the Poisson distribution inside of the program in addition to the EAS photons from the input ESAF file. The background photons were distributed homogeneously across the matrix of the TUS photodetector. The input EAS and background photons are ordered in time before of processing and elaborating of the output PMT current in the every pixel.

There are three mode of the TUSSIM operation: (i) input flux of photons which consists of the EAS and background photon mixure ordered in time, (ii) input flux of photons which consists of the pure EAS photons without of background photons and (iii) input flux of photons which consists of background photons without EAS photons. Every photon on the PMT cathode forms the pulse of the anode current with an amplitude that is propotional to PMT amplification $G(c l, p x)$ where $c l$ is the cluster number and $p x$ is the pixel number in the cluster. The anode current exponentially decreased according to $R \cdot C$ value where $R$ and $C$ are the resistivity and capacity of the output circuit respectively. The value $R \cdot C$ is equal to $0.6 \mu s$ that gives $\sim 0.37$ reduction of anode current at time step $\Delta t$.

Amplification coefficients are reading from the calibration table file $G(c l, p x)$ that was obtained by dedicated PMT measurements. Distribution of the PMT amplification coefficients is presented in Fig. 3. As it will be shown the wide range of the PMT amplification coefficients is a big trouble that essentionally reduce the trigger efficiency due to formation the faked background triggers. The TUS simulation was done for two cases: with the "ideal" PMTs at $G(c l, p x) \equiv 1$ and with the "real" PMTs taken from the calibration table. 


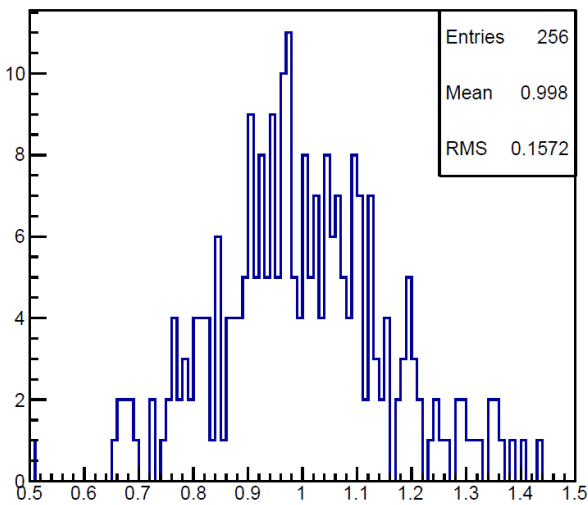

Figure 3: Distribution of the PMT amplification coefficients

\section{The TUS trigger efficiency simulation}

As it was mention above the TUS trigger rate has to be at the level of $1 \div 2$ triggers per minute and it leads to necessity to simulate more than $10^{6}$ events at each background level to evaluate trigger efficiency. It needs a great CPU time and do this approach is complicated. Way out was found by the separate generation of files with the S-sum values with a different level of the background radiation values and without of the EAS events and S-sum files with "ideal" PMTs without of the background. The size of background files corresponds to 1015 millons EAS events. The S-sum files of EAS events without background photons were produced similarly in $0.4 \cdot 10^{20}$ to $3.0 \cdot 10^{20} \mathrm{eV}$ energy range. Simultaneous analyses of the background S-sum files and EAS ones gives a possibility to increase a speed of the trigger efficiency study in 100-1000 times.
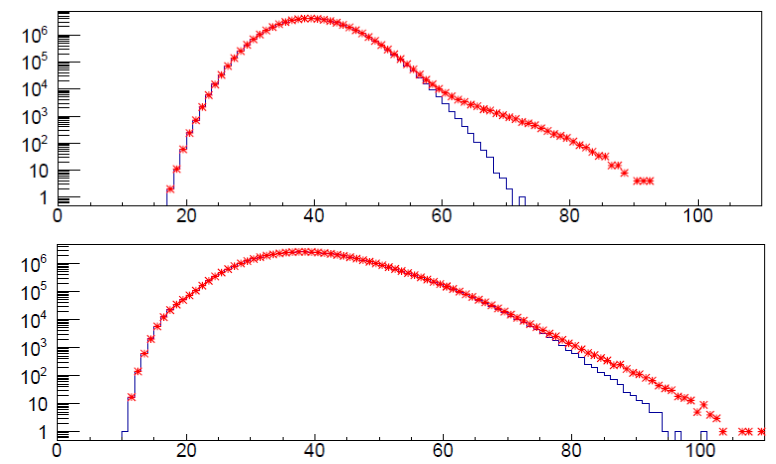

Figure 4: Sliding sum distributions for "ideal" PMTs (top) and "real" PMTs (bottom)

The statistical security of the trigger efficiency evaluation for the every EAS event was increased at the sufficient level by the its simulation together with the different $100 \div$ 500 statistically independent background S-sum files. The EAS flux at the $10^{20} \mathrm{eV}$ is exeptionally low $\sim 1$ event $/\left(100 \mathrm{~km}^{2} \cdot 100\right.$ years $)$ and the TUS detector will take mainly the faked events created by the atmospheric background radiation. It is important to provide an efficient trigger from the real EAS event also. In Fig. 4 the S-sum value distributions for "ideal" PMTs and "real" PMTs presented at the $10 \%$ of the avarage background level with the EAS events in the energy range $(0.5 \div 1.0) \times 10^{20} \mathrm{eV}$ on top of it. Obviously the 
trigger efficiency decreases dramatically in the case of the $16 \%$ amplification factor range of the PMTs that means practical impossibility for taking data with the Moon above the horizon. Presently there is an activity for on-line unification of the PMT output signals and the following simulation assumes mostly this case.

The TUS trigger rate (trigger of the level 2) depends on the trigger 1 threshold value and a requirement topology - the number of $M$ consecutive maps. The TUS trigger rate dependence is presented in Fig. 5 for the $10 \%$ and $20 \%$ of the avarage background level and the energy ranges $(0.5 \div 1.0) \times 10^{20} \mathrm{eV}$ and $(1.0 \div 1.5) \times 10^{20} \mathrm{eV}$ respectively. Different colors (green, red ...) correspond to the different trigger topologies: $M=2,3,4,5$. The upper gently sloping set of the histrgrams was obtaned with the EAS and background photons, the steeply-falling histograms correspond to the background photons without EAS ones. It was found that trigger efficiency is somewhat higher at $M=5$ topology and for example the trigger of the level 2 will be $T \sim 58$ and $T \sim 101$ for the trigger 1 threshold values in case of the $10 \%$ or $20 \%$ of the avarage background level correspondingly.
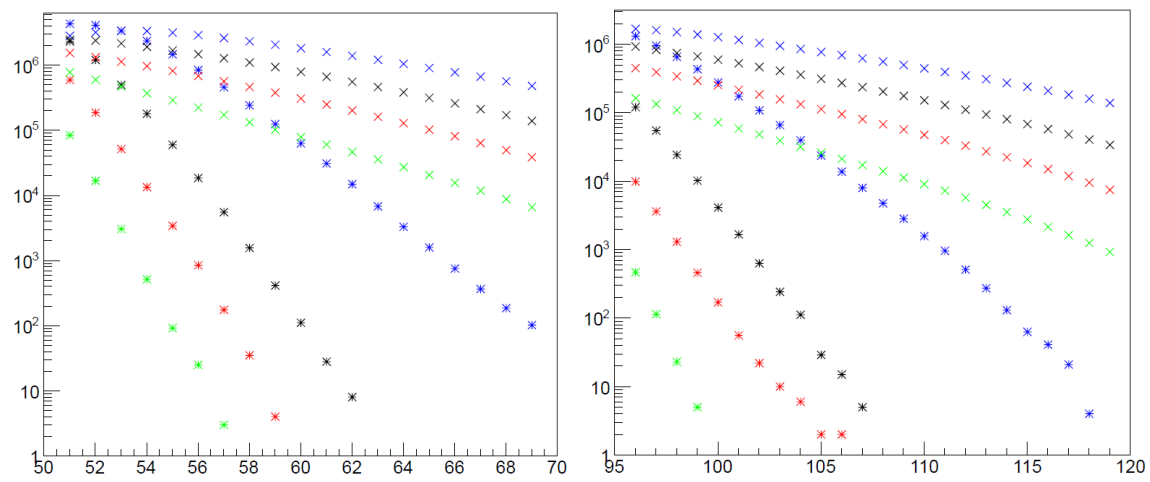

Figure 5: The TUS trigger rate per minute dependence of the trigger 1 treshold values. Simbols $\times$ and $*$ correspond to the cases with and without EAS photons. The background level is $10 \%$ (left) and $20 \%$ (right) of the avarage value.

To provide TUS trigger rate at the level of the 1 event per minute, the trigger 1 threshold value dependence of the avarage background light value was calulated and is shown in Fig. 6 for the different topologies of the level 2 trigger (left panel). It may be approximated by the power expression

$$
T(B g)=p_{\circ}+p_{1} \cdot B g+p_{2} \cdot B g^{2}
$$

where $B g$ is the backgrond light flux. Parameters $p_{i}$ of expession (4.1) were obtained by fit of this histograms and presented in the Table 1. Efficiency dependence of the threshold value at the $20 \%$ background and different energies are presented in the right panel of Fig. 6 .

\begin{tabular}{|c||c|c|c|}
\hline$M$ & $p_{\circ}$ & $p_{1}$ & $p_{2}$ \\
\hline \hline 3 & $11.74 \pm 3.1$ & $(2.58 \pm 0.24) 10^{-4}$ & $(-0.0038 \pm 0.003) 10^{-7}$ \\
\hline 4 & $10.41 \pm 3.1$ & $(2.54 \pm 0.24) 10^{-4}$ & $(-0.0037 \pm 0.003) 10^{-7}$ \\
\hline 5 & $8.62 \pm 3.1$ & $(2.49 \pm 0.24) 10^{-4}$ & $(-0.0038 \pm 0.003) 10^{-7}$ \\
\hline
\end{tabular}

Table 1: Results of fit of the histograms on the Fig, 6 

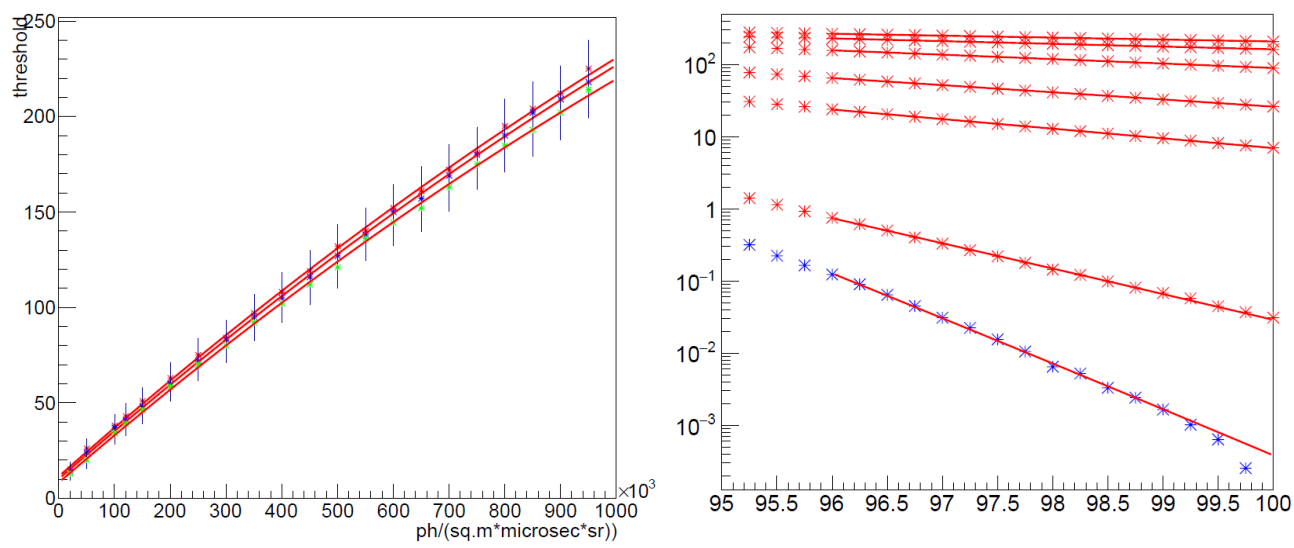

Figure 6: The trigger 1 threshold dependence of the background level (left). Efficiency dependence of the threshold value at the pure background (blue simbol) and at the different energies from $(0.5-1.0) \cdot 10^{20}$ to $(2.5-3.0) \cdot 10^{20} \mathrm{eV}$ (red simbols on the right panel). Curves are the power fits.

\section{Results of the TUS experiment simulation}

It needs to simulate the TUS background light level variation during the flight that depends on the mutual location of the satellite in orbit and the Sun and the Moon including the moon phase and its zenith angle in the satellite local coordinate system. The solarsinchronized orbit was simulated at the $500 \mathrm{~km}$ latitude for definiteness that means that the Sun is always in the orbital plane. The moon orbital plane around the Earth is coincide with the earth orbital plane around the Sun for simplicity. Duration of the turnover of the satellite was taken 90 minutes. In according to the EUSO assumption [9] the UHECR measurements are possible if the Sun locates at $10^{\circ}$ below of the horison in the satellite coodinate system. This decreases the effective the night data taking time to $\sim 38 \%$ for the satellite's orbit. The background atmospheric radiation is described by the empirical formula

$$
I_{M}=I_{\min }+\left(1.6 \cdot 10^{4} \cdot 10^{-0.4 \cdot\left(1.5 \cdot\left|\beta_{M}\right|+4.3 \cdot 10^{-2} \cdot \beta_{M}^{4}\right)}\right) \cos \theta_{M}
$$

where $\theta_{M}$ and $\beta_{M}$ are the zenith angle and the phase of the Moon respectively and $I_{\min }$ is the minimal background radiation $\sim 2 \cdot 10^{5} \mathrm{ph} /\left(\mathrm{sr} \cdot \mu_{\mathrm{s}} \cdot \mathrm{m}^{2}\right)$.

Experimental data of UHECR measurement in the region of GZK cutoff at $E \geq 3 \cdot 10^{19}$ $\mathrm{eV}[10]$ is fitted by the expression

$$
\lg \left(E^{3} J(E)\right)=37.74+(4.2-3.0) \cdot(19.41-\lg (E))
$$

where $J$ is the UHECR flux. Taking into account this flux the expected TUS statistics may be evaluated. This is shown in the Fig. 7 after 5 years of data collection from $500 \mathrm{~km}$ solarsinchronized orbit with $30 \%$ duty cycle taking into account a variation of the background light intensity during the space flight.

\section{Conclusion}

The TUS detector is integrated at the satellite Lomonosov and is scheduled for launch- 

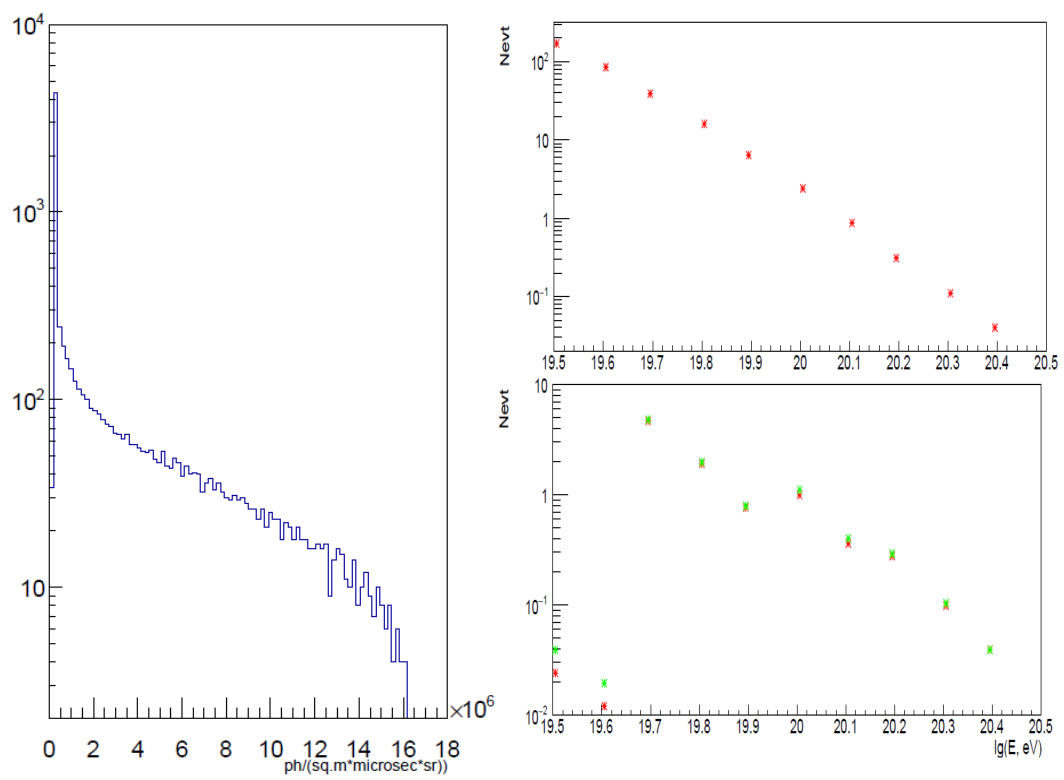

Figure 7: The TUS operation time in arbitrary units at the different background (left). The expected statistics for 5 years data taking: incoming flux (upper right), TUSSIM simulation (bottom right). The simbols $*$ and $*$ corresponds to $M=3$ and $M=5$ trigger topologies.

ing in late 2015. The TUSSIM simulation program takes into account most of peculiarities of the detector. In five years of operation in space the TUS exposure will be of the largest ground-based detectors to get a few UHECR events.

Acknowledgement: The authors thank TUS collaborators B. Khrenov, P. Klimov, S. Sharakin and I.V. Yashin for supporting of this work. This activity was partially supported by RFBR research project No. 15-02-05498 A.

\section{References}

[1] B. A. Khrenov et al., Nucl. Phys. B (Proc. Suppl.) 112 (2002) 115.

B. A. Khrenov et al., Phys. Atom. Nucl. 67(11) (2004) 2058.

[2] Tsunesada Y 2013 Proc. 33rd Int. Cosmic Ray Conf. Rio de Janeiro (2013) Abbasi R U et al. 2015 Astropart. Phys. 64 49-62 (Preprint arXiv:1408.1726)

[3] L.G. Tkatchev et al., 34th, this conference, ID 0681.

[4] P. A. Klimov et al., 33rd ICRC Proc. Rio De Janeiro (2013) ID 0406, M I Panasyuk et al., http://arxiv.org/pdf/1501.06368v2.pdf.

[5] Abrashkin et al., Advances in Space Research 37 (2006) 1867.

[6] DeMarco and M. Pallavicini. ESAF: The EUSO Simulation and Analysis Framework, 2002.Internal note EUSO-SIM-ESAF-001-01.

[7] G. Garipov et al., Phys. Part. Nuclei Lett. 10(1) (2013) 49. A. Grinyuk, M. Slunecka at al., NIMA 763 (2014), pp. 604-609.

[8] Vedenkin N N et al. Journal of Experimental and Theoretical Physics 113 (2011) 781. M.I.Panasyuk et al., Proc.ECRS 2014.

[9] Montanet F. EUSO note. Duty cycle: moon and sun light effects, PӨpril 2004, CI.16.

[10] M.Roth for Auger collaboration. Proc. of UHECR, CERN, Geneva, 2012 